\title{
Dominant negative STAT3 suppresses the growth and invasion capability of human lung cancer cells
}

\author{
GUOTAI XU, CAI ZHANG and JIAN ZHANG \\ School of Pharmaceutical Sciences, Shandong University, Jinan 250012, P.R. China
}

Received February 5, 2009; Accepted July 2, 2009

DOI: 10.3892/mmr_00000178

\begin{abstract}
Signal transducer and activator of transcription 3 (STAT3) is constitutively activated in diverse human cancers, including human lung cancer. In this study, STAT3 $\beta$, a dominant negative (DN) form of STAT3, was used to block activated STAT3 in human lung cancer cells and to confirm the effects of DN STAT3 on lung cancer cell proliferation and invasion in vitro. The results showed that the pIRES-STAT $3 \beta$ plasmid is efficiently transfected into and overexpressed in human lung cancer cells. Overexpressed STAT $3 \beta$ specifically blocked STAT3 transcriptional activation, inhibiting the proliferation and augmenting the apoptosis of human lung cancer cells. This was associated with the down-regulation of the anti-apoptotic gene bcl-xl and the cell cycle gene cyclin D1. Additionally, the invasive activity of A549 and PG cells was significantly inhibited by overexpressed STAT3 $\beta$, and was accompanied by a decrease in matrix metalloproteinase- 2 activation. These findings suggest that interfering with the DN STAT3, STAT3 $\beta$, may induce potent antitumor activity. STAT3 $\beta$ is therefore a potential candidate for the treatment of human lung cancers with high metastasic ability.
\end{abstract}

\section{Introduction}

Lung cancer is the leading cause of cancer mortality worldwide. Previous studies have indicated that increased lung cancer risk is associated with smoking, air pollution, environmental factors and oncogenes $(1,2)$. Over the last two decades, the number of smokers has dramatically increased in China, and a peak in the incidence of lung cancer is anticipated. Nearly $70 \%$ of patients suffering from lung cancer present with locally advanced or metastatic disease at the time of diagnosis (3), limiting treatment options.

Correspondence to: Dr Jian Zhang, Institute of Immunopharmacology and Immunotherapy, School of Pharmaceutical Sciences, Shandong University, 44 Wenhua West Road, Jinan 250012, P.R. China.

E-mail: zhangj65@sdu.edu.cn

Key words: signal transducer and activator of transcription 3, dominant negative, lung cancer, invasion
Many factors are involved in the occurrence and development of malignancies. In mammals, the JAK/STAT pathway is the main signaling pathway for various cytokines and growth factors (4). Cumulative evidence shows that the signal transducer and activator of transcription 3 (STAT3), a member of the STAT family, is constitutively activated in diverse human cancers, including lung, breast, head and neck, and hepatocellular carcinoma, as well as hematopoietic tumors (5-9). STAT3 has been implicated in the processes of cell proliferation, differentiation and apoptosis, and is associated with tumor invasion and metastasis $(10,11)$ and the negative regulation of innate and adaptive immune responses (12).

STAT3 activation is frequently observed in human lung cancer. Most patients diagnosed with lung cancer are not candidates for surgical resection due to the highly metastatic properties of the disease. Invasion-related proteins, in particular matrix metalloproteinases (MMPs), play a significant role in tumor invasion (13-15). Evidence indicates that the JAK/STAT3 signaling pathway may directly activate MMP promoters (11). The relationship between STAT3 activation and lung cancer cell invasion is unknown. In previous studies, we showed that blocking STAT3 using the STAT3-decoy ODN significantly inhibited the proliferation of PG and A549 cells in vitro (16), and dramatically inhibited A549 tumor growth in xenografted nude mice by inducing apoptosis or cell cycle arrest (17). In the present study, to investigate whether STAT3 is involved in lung cancer invasion, a dominant negative (DN) STAT3 was used.

STAT3 $\beta$ is a naturally-occurring splice variant of STAT3 (18) that lacks an intrinsic activation domain and has higher DNA-binding activity than STAT3. In the present study, STAT3 $\beta$ was used as a dominant negative form of STAT3 $(12,19)$, and was transiently transfected into A549 and PG cells. Cell proliferation, apoptosis and invasion were then analyzed.

\section{Materials and methods}

Cell lines and cell culture. The human non-small cell lung cancer A549 cell line and the human pulmonary giant cell carcinoma PG cell line were obtained from the American Type Culture Collection (Rockville, MD, USA), conserved at our laboratory and cultured in RPMI 1640 medium (Gibco BRL, Grand Island, NY, USA) supplemented with $10 \%$ heatinactivated FBS (Gibco), $100 \mathrm{IU} / \mathrm{ml}$ penicillin and $100 \mu \mathrm{g} / \mathrm{ml}$ streptomycin in $5 \% \mathrm{CO}_{2}$ at $37^{\circ} \mathrm{C}$. 
Transfection of dominant negative STAT3. The pIRES-EGFP (empty vector) and pIRES-STAT3 $\beta$ (DN STAT3) plasmids were kindly provided by Dr Yu Hua of the Beckman Research Institute (Duarte, CA, USA). All plasmid preparations were propagated in Escherichia coli (Tiangen, Beijing, China) and purified using the Endo-Free Plasmid Maxi kit (Qiagen, Hilden, Germany). Lipofectamine ${ }^{\mathrm{TM}} 2000$ (Invitrogen Life Technologies, Carlsbad, CA, USA) was used according to the manufacturer's instructions. The most efficient transfection was obtained using a Lipofectamine 2000 to DNA ratio of 2.5:1 $(\mu \mathrm{l}: \mu \mathrm{g})$. Subsequently, Western blotting was used to detect STAT3 $\beta$ protein levels in the transfected cells.

Luciferase reporter gene assay. As described previously (8), A549 cells were plated at density of $2 \times 10^{4}$ cells/well in 96-well plates (Costar) and cultured for $24 \mathrm{~h}$, then $0.05 \mu \mathrm{g}$ DN STAT3 or control vector was transiently co-transfected with $0.05 \mu \mathrm{g}$ pGL3-STAT3-TK-luc reporter gene plasmid or pGL3TK-luciferase control reporter construct. pRL-TK plasmid $(0.01 \mu \mathrm{g})$ (Promega, Madison, WI, USA) expressing Renilla luciferase was used to normalize for transfection efficiency. After 36 h, cells were washed and lysed, and a Dual-Glo ${ }^{\mathrm{TM}}$ Luciferase Assay System (Promega) was used to determine luciferase activity according to the manufacturer's protocol. The pGL3-STAT6-TK-luciferase reporter gene was used to investigate the specificity of DN STAT3 in A549 cells.

Cell proliferation assay. Lung cancer cells $\left(8 \times 10^{4}\right)$ were seeded into a 24-well plate and cultured for $24 \mathrm{~h}$, then transfected with $0.3 \mu \mathrm{g}$ DN STAT3 or vector for $6 \mathrm{~h}$ according to the method described above. The medium was replaced by fresh RPMI 1640 medium supplemented with 10\% FBS without antibiotics and cultured for another $6 \mathrm{~h}$. Subsequently, the cells were detached by trypsin-EDTA ( 0.25 and $0.02 \%$, respectively), and $6 \times 10^{3}$ cells/well in $100-\mu 1$ aliquots were seeded in 96-well plates. After being cultured for 24, 48 and $72 \mathrm{~h}, 10 \mu \mathrm{l}$ of Cell Counting Kit-8 (CCK-8) solution (Dojindo, Kumamoto, Japan) was added to each well (100 $\mu 1$ medium) at the indicated times and incubated for another $2 \mathrm{~h}$ at $37^{\circ} \mathrm{C}$. Cell growth was determined using a microplate reader at $450 \mathrm{~nm}$, with $630 \mathrm{~nm}$ as the reference wavelength. The inhibition rate was calculated as follows: inhibition rate $(\%)=\left(\mathrm{OD}_{\text {control }}-\mathrm{OD}_{\mathrm{T}}\right) / \mathrm{OD}_{\text {control }} \times 100 \%$.

Western blotting. Western blotting was used to identify STAT3 and its downstream gene expression levels. Anti-STAT3, antibcl-xl, anti-cyclin D1 and anti- $\beta$-actin antibodies, as well as horseradish peroxidase-conjugated secondary antibody, were purchased from Cell Signaling Technology (Beverly, MA, USA). As previously reported $(16,17)$, cells were lysed and whole cell extracts $(30 \mu \mathrm{g} / \mathrm{lane})$ were separated by SDS-PAGE and transferred to PVDF membranes (Millipore, Bedford, MA, USA). After being blocked with 5\% (w/v) skim milk, the membranes were incubated with primary antibody at a dilution of 1:1000, and then incubated with horseradish peroxidaseconjugated secondary antibody (1:1000). Protein levels were visualized using enhanced chemiluminescence (Pierce Biotechnology, Inc., Rockford, IL, USA) and exposed to X-ray film (Kodak). The bands were examined by densitometry using AlphaEaseFC software, with standardization of each band to its corresponding loading control.
Cell apoptosis assay. Forty-eight hours after transfection with DN STAT3 or control vector, cells were released, washed and resuspended in $300 \mu 1$ Annexin V binding buffer, and subsequently stained with Annexin V-FITC (Jingmei, Shenzhen, China) and propidium iodide (PI) (Jingmei) according to the manufacturer's instructions. Data acquisition and analysis were performed by FACS using CellQuest software. For each condition, 10,000 cells were evaluated. Positive staining for Annexin V indicated cells undergoing early apoptosis, whereas Annexin V/PI staining indicated late apoptosis.

Cell invasion assay. Matrigel (BD Biosciences, Mississauga, Ontario, Canada) was diluted with cold serum-free RPMI 1640 medium (1:9), then $50 \mu \mathrm{l}$ were applied to the $8-\mu \mathrm{m}$ pore size polycarbonate membrane filters of a Boyden chamber (Costar, Cambridge, MA, USA) and solidified at $37^{\circ} \mathrm{C}$ for $4 \mathrm{~h}$. The cells were seeded into the upper compartment of the Boyden chamber at a density of $1 \times 10^{5}$ cells $/ 200 \mu \mathrm{l}$ of serum-free medium. The bottom chamber contained standard medium with $10 \%$ FBS. As described previously (2), after incubation at $37^{\circ} \mathrm{C}$ for $24 \mathrm{~h}$, cells that had invaded the Matrigel and attached to the lower surface of the filter were fixed with methanol and stained with $0.1 \%$ crystal violet. The number of cells was counted in eight randomized fields under a light microscope at a magnification of $x 400$.

Zymography of matrix metalloproteinases. Zymographic analysis of gelatinase activity in secreted medium was performed in $10 \%$ SDS-PAGE containing $0.1 \%$ gelatin (2). Cells $\left(2 \times 10^{5} /\right.$ well) in $300 \mu$ l serum-free medium were seeded into a 24-well plate. After $24 \mathrm{~h}, 60 \mu \mathrm{l}$ of the supernatants were collected and mixed with $20 \mu \mathrm{l} 4 \mathrm{X}$ loading buffer for electrophoresis. Following electrophoresis, the gel was washed twice with $2.5 \%$ Triton X-100 for $45 \mathrm{~min}$ to remove the SDS, and then incubated at $37^{\circ} \mathrm{C}$ in $50 \mathrm{mmol} / \mathrm{l}$ Tris- $\mathrm{HCl}$ buffer $(\mathrm{pH}$ 7.6) containing $5 \mathrm{mmol} / 1 \mathrm{CaCl}$ and $0.02 \% \mathrm{ZnCl}$. After $24 \mathrm{~h}$, the gel was stained with 0.1\% Coomassie Brilliant Blue R-250 (Bio-Rad, Hercules, CA, USA) for $4 \mathrm{~h}$ and briefly de-stained in $10 \%$ acetic acid and $30 \%$ methanol. Pre-stained SDS-PAGE protein standards (Tiangen) were used to estimate molecular mass. The experiment was conducted three times consecutively.

Statistical analysis. All data were expressed as the means \pm standard deviation (SD) of three or more individual experiments. Differences in mean values between two groups were tested by the Student's t-test. P-values $<0.05$ were considered statistically significant.

\section{Results}

STAT3 $\beta$ is overexpressed in human lung cancer cells. To confirm whether DN STAT3 can be efficiently transfected into human lung cancer cells and overexpressed, A549 and PG cells were transfected with DN STAT3 and control vector, respectively, and then the cell lysates were prepared and STAT3 protein levels were analyzed by Western blotting. As shown in Fig. 1, in cells transfected or not transfected with control vector, the expression levels of STAT3 were generally present at similar concentrations; STAT3 $\beta$ isoform was overexpressed in cells transfected with DN STAT3, but not in 
A.

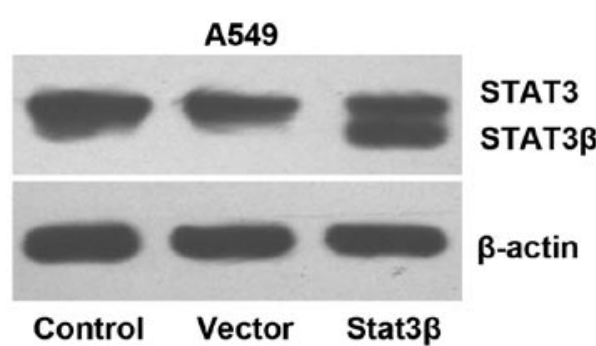

B.

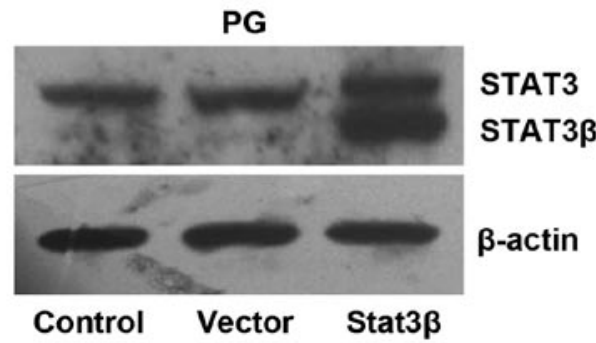

Figure 1. STAT3B was overexpressed in human lung cancer cells treated with dominant negative (DN) STAT3. Western blotting was used to analyze the expression levels of STAT3 and STAT3B in A549 (A) and PG (B) cells transfected with DN STAT3. Representative results of at least three independent experiments are shown.

cells transfected or not transfected with control vector. These results confirmed that DN STAT3 plasmid was efficiently transfected into human lung cancer cells, and that STAT3 $\beta$ was overexpressed. Therefore, this protocol for transfecting DN STAT3 vector was used in subsequent experiments.

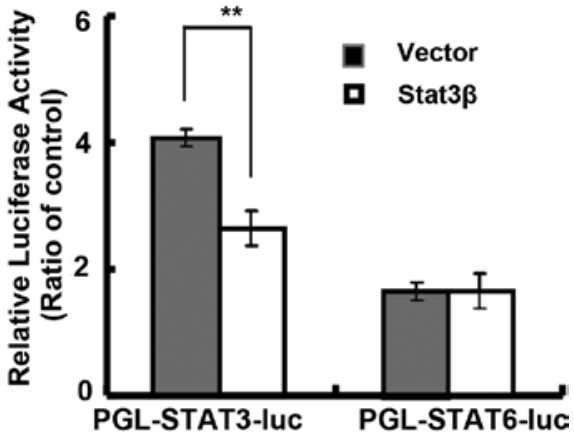

Figure 2. Dominant negative (DN) STAT3 suppressed the transactivation of STAT3 in A549 cells. As described in Materials and methods, A549 cells were co-transfected with control vector or DN STAT3 and pGL3-STAT3-TKluciferase or pGL3-STAT6-TK-luciferase construct, while pRL-TK was used to normalize for transfection efficiency. After $36 \mathrm{~h}$, a Dual-Glo ${ }^{\text {TM }}$ Luciferase Assay System was applied to determine luciferase activity. Data represent the mean \pm SD of three independent experiments. The results were statistically significant $\left({ }^{* *} \mathrm{P}<0.01\right.$; Student's t-test).

Transcription effect of STAT3 is suppressed by DN STAT3. We went on to investigate the influence of overexpressed STAT3 $\beta$ on STAT3 transcriptional activation by the Luciferase Reporter Assay System. The results showed that overexpressed STAT3 $\beta$ remarkably reduced the transcription activity of STAT3 by $\sim 35 \%$ compared with cells transfected with control vector (Fig. 2). Simultaneously, the pGL3-STAT6-TK-luciferase reporter gene was used to identify the specificity of DN STAT3. The results indicated that DN STAT3 did not affect the transactivation of STAT6 in A549 cells (Fig. 2B), which confirmed the specificity of DN STAT3 for affecting STAT3 transactivation in A549 cells. These observations suggest that STAT3 $\beta$

A.
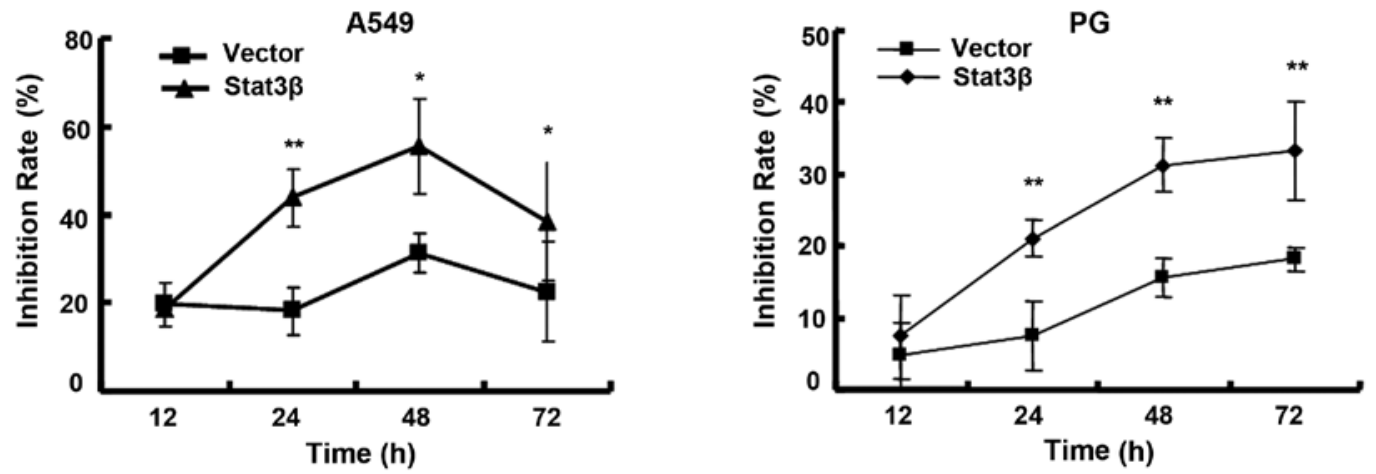

B.
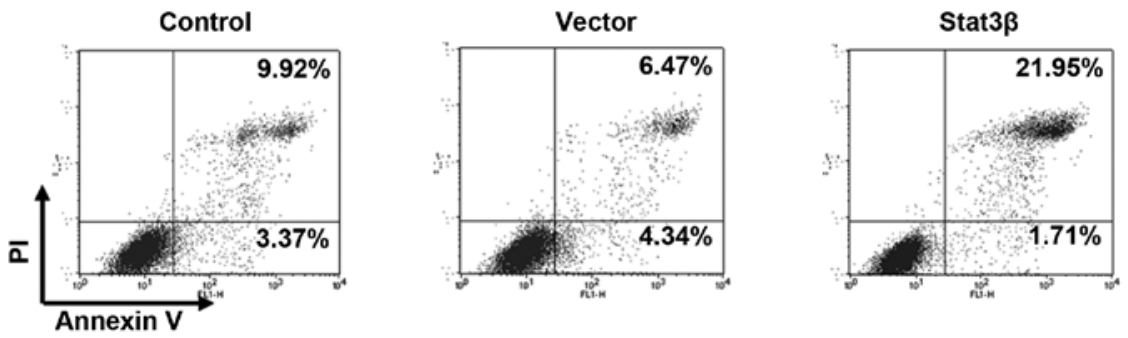

Figure 3. Proliferation and apoptosis of human lung cancer cells treated with dominant negative (DN) STAT3 in vitro. (A) The proliferation potency of cells overexpressing STAT3 $\beta$ was evaluated using CCK- 8 . Each value is the mean \pm SD of four independent experiments. The results were statistically significant $\left({ }^{*} \mathrm{P}<0.05,{ }^{* *} \mathrm{P}<0.01\right.$; Student's t-test). (B) After $48 \mathrm{~h}$, the apoptotic rate of cells transfected with DN STAT3 or control vector was analyzed by flow cytometry using Annexin V-FITC and propidium iodide. Values are representative of three independent experiments. 
A.

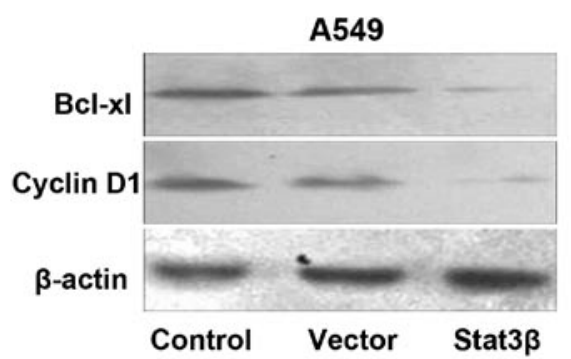

B.

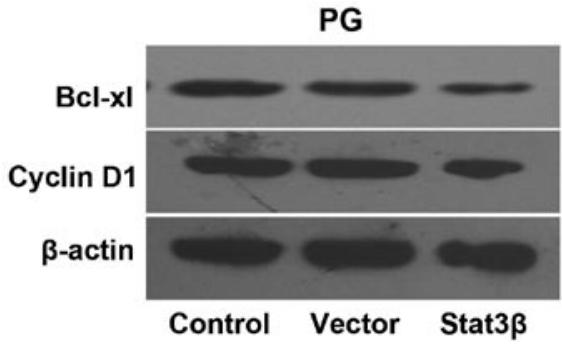

Figure 4. Dominant negative (DN) STAT3 down-regulated anti-apoptotic and cell cycle-related genes. A549 (A) and PG (B) cells transfected with DN STAT3 or control vector were harvested at $48 \mathrm{~h}$. Protein levels of bcl-xl and cyclin D1 were characterized by Western blot analysis. $\beta$-actin was used as a loading control to normalize for overall protein concentration. Data are representative of three independent experiments.

overexpression inhibits STAT3 transcriptional activity via competitive DNA-binding.

DN STAT3 suppressed proliferation and promoted apoptosis of human lung cancer cells in vitro. Since STAT3 is implicated in the control of cell proliferation, differentiation and apoptosis, the effect of STAT3 $\beta$ expression on the growth of A549 and PG cells was investigated using CCK-8. After transfection with DN STAT3 or control vector, cells were cultured for 12,
24,48 or $72 \mathrm{~h}$, then $10 \mu \mathrm{l}$ of CCK-8 was added to each well and the cells were incubated for another $2 \mathrm{~h}$. Fig. 3A shows that the overexpression of STAT $3 \beta$ conspicuously suppressed the proliferation of A549 and PG cells, with a maximum inhibition rate of $55 \%(\mathrm{P}<0.01)$ or $33 \%(\mathrm{P}<0.01)$, respectively. At the same time, the rate of apoptosis was evaluated using Annexin-V/PI staining. The results revealed that the apoptotic rate was significantly elevated in DN STAT3-transfected A549 cells (23.66\%) (Fig. 3B) compared to blank vector-treated cells $(10.81 \%)$ or control cells $(13.29 \%)$.

Overexpressed STAT3 $\beta$ down-regulated anti-apoptotic and cell cycle-related genes. As a transcription factor, STAT3 controlled many genes, including anti-apoptotic and cell cycle genes. Western blotting was used to investigate the effects of DN STAT3 on the cell cycle gene cyclin D1 and the antiapoptotic gene bcl-xl. Fig. 4 shows that the disruption of STAT3 signaling by the overexpression of STAT3 $\beta$ significantly reduced cyclin D1 and bcl-xl expression levels in DN STAT3-treated human lung cancer cells.

Inhibition of DN STAT3-mediated invasion by the downregulation of matrix metalloproteinase-2 activation. As previously reported, STAT3 was associated with malignant invasion $(10,11)$. To clarify the direct effects of STAT3 $\beta$ on the invasive ability of human lung carcinoma cells, the highly invasive A549 and PG cell lines were treated with DN STAT3, and then their invasive ability was analyzed using the Transwell assay. As shown in Fig. 5, a significant reduction in the number of invasive cells was observed in cells treated with DN STAT3. Compared to the control cells, the invasive ability of the cells transfected with DN STAT3 was decreased by $>30 \%(\mathrm{P}<0.01)$.

Matrigel degradation is necessary for cellular invasion, and MMP-2 is particularly involved in lung cancer cells (15). To clarify whether MMP-2 is involved in the inhibition of
A.

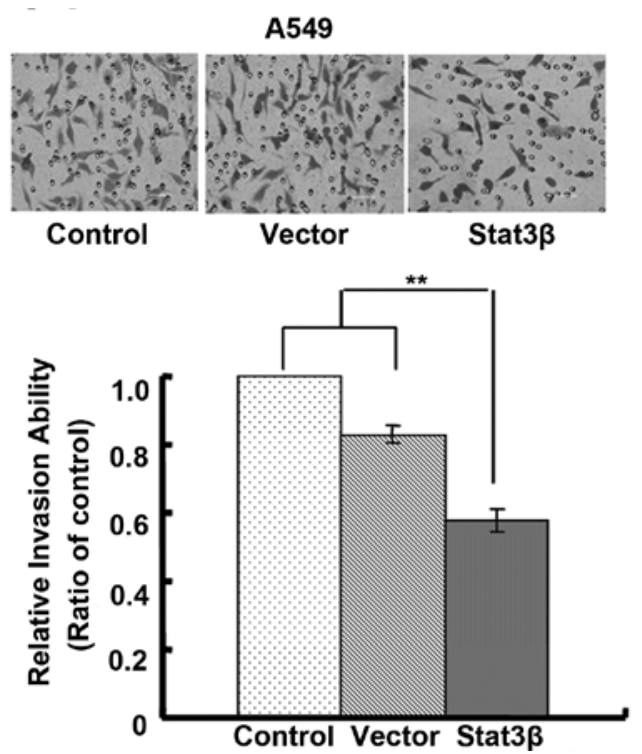

B.

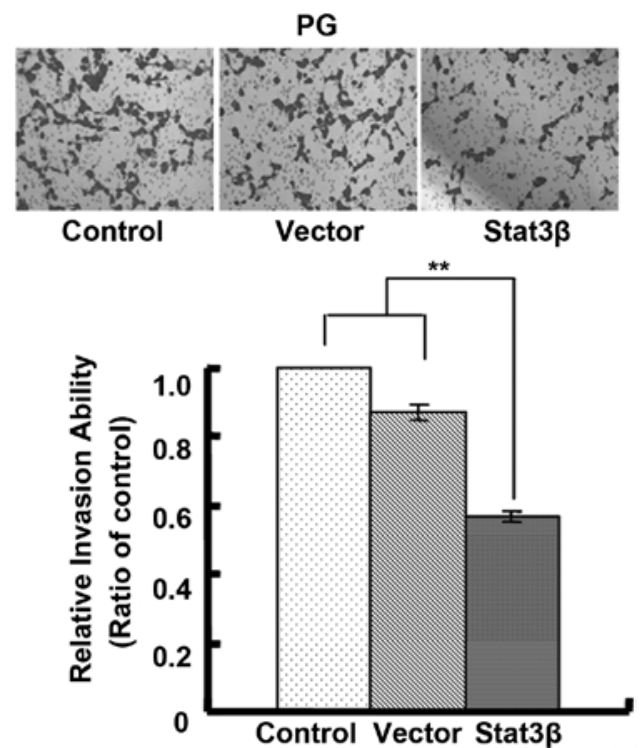

Figure 5. Dominant negative (DN) STAT3 inhibited the invasion of human lung cancer cells. The Transwell assay was used to evaluate the effect of DN STAT3 on the invasive capability of A549 (A) and PG (B) cells. Cells that invaded the lower surface of the filter were fixed with methanol and stained with $0.1 \%$ crystal violet. The number of cells in eight randomly-selected fields was counted under a light microscope at x400 magnification. Representative results of four independent experiments are shown. The results were statistically significant $\left({ }^{* *} \mathrm{P}<0.01\right.$; Student's t-test). 
A.

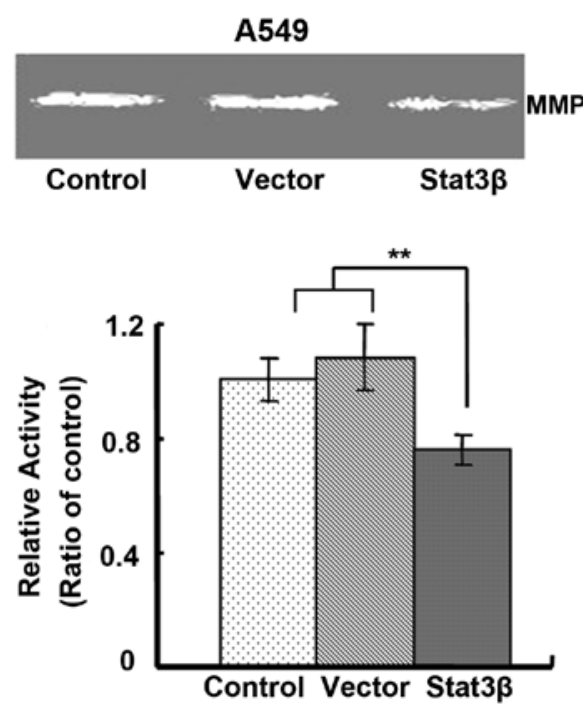

B.
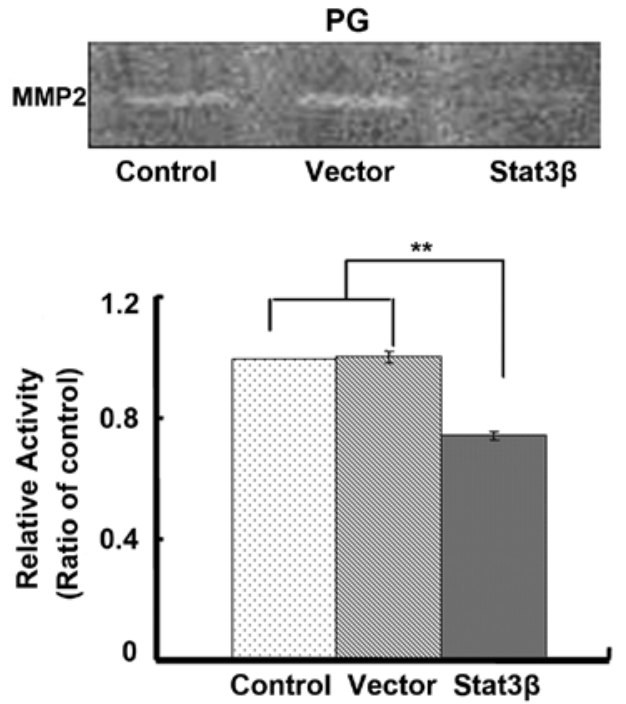

Figure 6. Dominant negative (DN) STAT3-mediated inhibition of MMP2 activity in human lung cancer cells. As described in Materials and methods, MMP-2 activity in the supernatant of A549 (A) and PG (B) cells treated with DN STAT3 was measured using zymographic analysis. Representative results from four independent experiments are shown. The results were statistically significant $\left({ }^{* *} \mathrm{P}<0.01\right.$; Student's t-test).

invasion mediated by overexpressed STAT3 $\beta$, MMP- 2 activity was investigated using gelatin zymography under serum starvation conditions. As shown in Fig. 6, the overexpression of STAT3 $\beta$ significantly reduced MMP-2 activity by almost $30 \%$ in comparison with the control cells. The impact of STAT3 $\beta$ on MMP-9 activity could not be assessed due to the extremely low expression of MMP-9 in A549 and PG cells, even in the absence of STAT3 $\beta$.

\section{Discussion}

STAT3 is a latent cytoplasmic transcription factor that has been shown to be oncogenic when constitutively activated. We and others have demonstrated that STAT3 is constitutively activated with high frequency in various human tumor cell lines $(5-9,16,17)$, and that STAT3 activation is associated with cancer cell growth and survival. In addition, persistent activation of STAT3 promotes tumor angiogenesis and metastasis $(11,21,22)$. Recent studies have shown that STAT3 signaling plays a critical role in tumor cell immune evasion by negatively regulating cellular and innate immune responses $(12,23)$. Interference by the constitutive STAT3 signaling pathway reverses chemotherapy resistance, reduces tumor growth and metastasis and induces cancer cell death. STAT3 is therefore a promising candidate for cancer therapy.

We previously conducted a study exploring the relationship between the activation of STAT3 and lung cancer $(16,17)$. However, the biological significance of STAT3 activation in lung cancer, including tumor invasion, remains to be fully elucidated. In the present study, DN STAT3-STAT3 $\beta$ strategy was used as gene therapy for the A549 and PG lung cancer cell lines. Our findings indicate that overexpressed STAT3 $\beta$ specifically represses STAT3 transactivity and down-regulates target genes such as cyclin D1 and bcl-xl. STAT3 $\beta$ may also inhibit cell proliferation and induce cell apoptosis, and may suppress human lung cancer cell invasion by decreasing the activity of MMP-2 in vitro. Based on our findings, DN STAT3 may be a promising candidate for the treatment of lung cancer with high invasive and metastasic properties. Additionally, as DN STAT3 is efficiently transfected and consistently overexpressed in tumor cells, it may be used for clarifying the biological significance of STAT3 in lung cancer cells in vitro and in vivo.

\section{Acknowledgements}

This work was supported by grants from the Natural Science Foundation of China (nos. 30671901 and 30571696) and the Ministry of Science and Technology of China (863 program no. 2007AA021000; 973 program nos. 2004CB518807, 2004CB520804 and 2006CB504303).

\section{References}

1. Lee GY, Jang JS, Lee SY, et al: XPC polymorphisms and lung cancer risk. Int J Cancer 115: 807-813, 2005.

2. Shih JY, Tsai MF, Chang TH, et al: Tanscription repressor slug promotes carcinoma invasion and predicts outcome of patients with lung adenocarcinoma. Clin Cancer Res 11: 8070-8078, 2005.

3. Molina JR, Yang P, Cassivi SD, Schild SE and Adjei AA: Non-small cell lung cancer: epidemiology, risk factors, treatment, and survivorship. Mayo Clin Proc 83: 584-594, 2008.

4. Rawlings JS, Rosler KM and Harrison DA: The JAK/STAT signaling pathway. J Cell Sci 117: 1281-1283, 2004.

5. Alvarez JV, Greulich H, Sellers WR, Meyerson M and Frank DA: Signal transducer and activator of transcription 3 is required for the oncogenic effects of non-small-cell lung cancer-associated mutations of the epidermal growth factor receptor. Cancer Res 66: 3162-3168, 2006

6. Garcia R, Bowman TL, Niu G, et al: Constitutive activation of Stat 3 by the Src and JAK tyrosine kinases participates in growth regulation of human breast carcinoma cells. Oncogene 20: 2499-2513, 2001.

7. Leong PL, Andrews GA, Johnson DE, et al: Targeted inhibition of Stat3 with a decoy oligonucleotide abrogates head and neck cancer cell growth. Proc Natl Acad Sci USA 100: 4138-4143, 2003.

8. Sun X, Zhang J, Wang L and Tian Z: Growth inhibition of human hepatocellular carcinoma cells by blocking STAT3 activation with decoy-ODN. Cancer Letters 262: 201-213, 2008. 
9. Gouilleux-Gruart V, Gouilleux F, Desaint C, et al: STAT-related transcription factors are constitutively activated in peripheral blood cells from acute leukemia patients. Blood 87: 1692-1697, 1996.

10. Horiguchi A, Oya M, Shimada T, Uchida A, Marumo K and Murai M: Activation of signal transducer and activator of transcription 3 in renal cell carcinoma: a study of incidence and its association with pathological features and clinical outcome. J Urol 168: 762-765, 2002.

11. Xie TX, Wei D, Liu M, Gao AC, Ali-Osman F, Sawaya R and Huang S: Stat 3 activation regulates the expression of matrix metalloproteinase- 2 and tumor invasion and metastasis. Oncogene 23: 3550-3560, 2004

12. Kahari VM and Saarialho-Kere U: Matrix metalloproteinases and their inhibitors in tumour growth and invasion. Ann Med 31: 34-45, 1999.

13. Wang T, Niu G, Kortylewski M, et al: Regulation of the innate and adaptive immune responses by Stat-3 signaling in tumor cells. Nat Med 10: 48-54, 2004.

14. Azzam HS, Arand G, Lippman ME and Thompson EW: Association of MMP-2 activation potential with metastatic progression in human breast cancer cell lines independent of MMP-2 production. J Natl Cancer Inst 85: 1758-1764, 1993.

15. Chetty C, Bhoopathi P, Joseph P, Chittivelu S, Rao JS and Lakka S: Adenovirus-mediated small interfering RNA against matrix metalloproteinase-2 suppresses tumor growth and lung metastasis in mice. Mol Cancer Ther 5: 2289-2299, 2006.

16. Zhang X, Zhang J, Wei H and Tian Z: STAT3-decoy oligodeoxynucleotide inhibits the growth of human lung cancer via down-regulating its target genes. Oncol Rep 17: 1377-1382, 2007.
17. Zhang X, Zhang J, Wang L, Wei H and Tian Z: Therapeutic effects of STAT3-decoy oligodeoxynucleotide on human lung cancer in xenograft mice. BMC Cancer 7: 149-159, 2007.

18. Caldenhoven E, van Dijk TB, Solari R, et al: STAT3 $\beta$, a splice variant of transcription factor STAT3, is a dominant negative regulator of transcription. J Biol Chem 271: 13221-13227, 1996.

19. Niu G, Shain KH, Huang M, et al: Overexpression of a dominantnegative signal transducer and activator of transcription 3 variant in tumor cells leads to production of soluble factors that induce apoptosis and cell cycle arrest. Cancer Res 61: 3276-3280, 2001.

20. Jiang W, Sun R, Wei H and Tian Z: Toll-like receptor 3 ligand attenuates LPS-induced liver injury by down-regulation of toll like receptor 4 expression on macrophages. Proc Natl Acad Sci USA 102: 17077-17082, 2005.

21. Wei D, Le X, Zheng L, et al: Stat 3 activation regulates the expression of vascular endothelial growth factor and human pancreatic cancer angiogenesis and metastasis. Oncogene 22: 319-329, 2003.

22. Xiong H, Zhang ZG, Tian XQ, et al: Inhibition of JAK1, 2/STAT3 signaling induces apoptosis, cell cycle arrest, and reduces tumor cell invasion in colorectal cancer cells. Neoplasia 10: 287-297, 2008.

23. Buettner R, Huang M, Gritsko T, et al: Activated signal transducers and activators of transcription 3 signaling induces CD46 expression and protects human cancer cells from complementdependent cytotoxicity. Mol Cancer Res 5: 823-832, 2007. 